\title{
Problemas éticos en las publicaciones científicas
}

Presidente de la Academia Chilena de Medicina. Editor Emérito de la Revista Médica de Chile.

HUMBERTO REYES B.

\section{Ethical problems in scientific publications}

Ethical problems have always been present in scientific publications. Since the founding of medical journals, in the XIX Century, until today they are a source of concern because one main purpose of medical scientific publications is to add new, reliable information that could guide or modify medical decisions and public health policies. Since 1997, Revista Médica de Chile has published several articles clarifying this situation and emphasizing the need to avoid ethical misbehavior. The present review reminds that the main sources of information dealing with publication ethics appear in the web sites of ICMJE, COPE and WAME. Misconduct have been detected in Revista Médica de Chile in a few cases of redundant publications, plagiarism, lack of recognition of conflicts of interest mainly with pharmaceutical companies, and one attempt of forging the publication of an article that had been previously rejected. In handling situations identified as ethical misbehavior, the editors of this journal have successfully followed rules established by COPE. This article reviews and reinforces recommendations to avoid ethical misbehavior in biomedical research and in manuscripts submitted for publication.

(Rev Med Chile 2018; 146: 373-378)

Key words: Bioethics; Clinical Trials Data Monitoring Committees; Conflict of Interest; Plagiarism; Scientific Misconduct.

"El centro de la Medicina es el enfermo y su principio rector es buscar siempre su bien; por lo tanto, la investigación biomédica debe beneficiarlo, protegerlo y no dañarlo"1.

\section{A} comienzos del siglo XVII se fundaron las primeras sociedades y academias científicas, para facilitar la comunicación entre los científicos y hacer respetar el derecho de autoría, tratando de eliminar el plagio y el robo intelectual. La necesidad de facilitar el contacto entre sus miembros condujo a la creación de revistas científicas, que se convirtieron en la vía preferida para la difusión de nuevas ideas y conocimientos y en repositorios de la información científica.

Han transcurrido tres siglos de crecimiento en la producción de documentos científicos, con una velocidad abismante desde la segunda mitad del siglo XX, pero no hemos resuelto un problema fundamental: si las revistas científicas surgieron, además de ser vehículos de difusión de las ciencias, como una respuesta al plagio o robo intelectual, y el proceso de editarlas es ahora una actividad profesional, ¿por qué los delitos científicos siguen siendo un problema inquietante?

La presión por publicar es un determinante crucial en la carrera de los investigadores porque de ello dependen sus posibilidades de obtener recursos para subsistir como profesionales, seguir investigando y ascender en el escalafón académico. Hay adagios de larga data que ayudan a explicar la mala conducta en las publicaciones científicas: "Lo que no se publica no existe", "Publish or perish" (Publicar o perecer), "Publicando se llega al paraíso académico". Todos pueden inducir al pecado... 
El presente artículo se basa en una conferencia dictada en la Academia Chilena de Medicina ${ }^{2}$, reiterando aquí algunos aspectos claves en la ética de las publicaciones científicas y enlistando artículos en la Revista Médica de Chile que ilustran el interés de los autores y los editores por difundir el tema ${ }^{3-17}$. Se destacan las publicaciones especializadas que dan más información sobre la ética de las publicaciones científicas, las recomendaciones para evitar sus transgresiones, la forma de investigarlas y las sanciones que corresponde aplicar ${ }^{18-21}$.

Las transgresiones a la ética científica son motivo de preocupación internacional por el daño que pueden ocasionar en el avance de los conocimientos científicos y en el ejercicio profesional médico. En 1992, el gobierno de los Estados Unidos de Norteamérica fundó una Oficina de Integridad de la Investigación (ORI) que acoge e investiga denuncias de posible mala conducta científica ${ }^{22}$. Esta oficina ha recibido treinta a cuarenta nuevas denuncias por año. Aunque al término de la investigación respectiva no todas resultaron ser fraudes, dan una imagen de la magnitud que pueden tener los delitos científicos.

\section{Ética de las publicaciones científicas y su importancia en medicina}

La ética de las publicaciones científicas es la ética de la investigación científica y de la creación intelectual, más la ética en el proceso editorial que se extiende desde que una revista recibe un manuscrito hasta que es publicado. Todo asienta en tres pilares: 1 . Honestidad = probidad, rectitud, honradez, decencia; 2 . Transparencia = claridad, sin ambigüedad, evidencia; y 3. Responsabilidad = confianza, "accountability" (capacidad de rendir cuentas).

Uno de los propósitos de las revistas médicas es influir en el pensamiento y el actuar clínico de sus lectores. El respeto de las normas éticas es indispensable para que sus publicaciones aporten beneficios reales y confiables. Por ello, es importante incluir en la educación profesional de pre y postítulo discusiones sobre la ética de la investigación científica y de sus publicaciones ${ }^{8}$. Las personas que intervienen en el proceso editorial (autores de manuscritos, sus revisores externos y los editores) deben actuar con buena fe, buen criterio y amparados en el sentido común.

\section{$\underline{\text { Requisitos éticos en las publicaciones médicas }}$}

Las normas de la investigación médica, cuyo cumplimiento debe ser exigido en todas las publicaciones a que dé lugar, están centradas en la "Declaración de Helsinki" sobre el respeto a los derechos humanos. El primer filtro para verificar su cumplimiento recae en los mismos autores $\mathrm{y}$ en las autoridades que aprobaron iniciar el estudio, seguido por un segundo filtro, que es el Comité de Ética de la Investigación que lo autorizó. Estos comités incluyen expertos en bioética, un asesor jurídico y un representante de la comunidad, y su estructura y metodología deben ser acreditadas por la autoridad sanitaria (Ministerio de Salud) ${ }^{17,23}$. Un tercer filtro recae en las revistas que reciben los manuscritos. Sus editores pueden solicitar a los autores una copia de la resolución del Comité de Ética que autorizó la investigación, particularmente si ellos o los revisores han detectado debilidades éticas en el trabajo en revisión.

Hay normas para las investigaciones en personas (pacientes y controles) y para el procesamiento de tejidos, células, humores o líquidos tisulares obtenidos en seres humanos. Ellas abarcan desde la toma de las muestras hasta su manejo y destino final. Las normas se extienden a la protección de quienes participan como ejecutores de la investigación. A las disposiciones y recomendaciones de carácter universal debe agregarse las normativas que imperan en cada país. En el caso de Chile, hay leyes que se refieren específicamente a los ensayos clínicos y, en algunos casos, se extienden a la investigación clínica en general (ejemplos: Leyes No 20.120, 20.584 y 20.850 -"Ley Ricarte Soto") 17,23 .

El International Committee of Medical Journal Editors (ICMJE) define los ensayos clínicos como "cualquier proyecto de investigación que asigne prospectivamente a una persona o grupos de personas a una intervención, con o sin una comparación simultánea o un grupo de control, para estudiar las relaciones entre una intervención relacionada con la salud y un resultado en la salud"18. Esta definición ha sido ampliamente aceptada, quedando en claro que no se refiere solamente a la investigación farmacológica o de equipos e instrumentos, sino que también aplica a las áreas de la psicología y la sociología, donde una encuesta o una entrevista profesional 
puede provocar una intervención sobre el comportamiento o la forma de pensar de los sujetos investigados.

La definición de "ensayos clínicos" no aplica a estudios epidemiológicos que recurren a bases de datos, sin contacto directo con las personas de donde proceden estos datos; este tipo de estudios puede calificarse como "investigación médica" o "investigación clínica”, pero no "ensayo clínico". Tampoco corresponde aplicarla a los estudios fisiopatológicos observacionales en que no se ejecuta ninguna intervención sobre los sujetos estudiados.

Una norma ética elemental es respetar el anonimato de las personas estudiadas, eliminando cualquier palabra o símbolo que permita identificarlas. Las fotografías son elementos que exigen un cuidado especial: desde hace más de una década se ha establecido que el rostro de las personas solo puede reproducirse en casos con interés clínico específico y con la autorización escrita y firmada por la persona o su representante legal, los que deben tener la oportunidad de conocer el documento en que aparecerá la fotografía, antes de que sea publicado. Obviamente, es inaceptable limitarse a cubrir los ojos de la persona fotografiada.

Existen también normas que regulan el manejo de animales de experimentación y los proyectos que los utilizan deben ser aprobados por comités de ética específicos para este campo de la investigación ${ }^{24}$.

\section{Ejemplos de aspectos éticos que suelen ocasionar problemas}

\section{¿Quiénes son los autores de un manuscrito?}

El ICMJE exige cumplir con cuatro requisitos para justificar la inclusión de un autor en un manuscrito, los cuales han sido consensuados internacionalmente ${ }^{18}$ :

\section{Tuvo una contribución sustancial en}

- La concepción y diseño del estudio o

- La adquisición de los datos o

- El análisis e interpretación de los datos; Y

2. Participó en la redacción del manuscrito o en su revisión crítica, con un aporte intelectual a este; $\mathrm{Y}$

3. Dio su aprobación a la versión final del manuscrito, que será publicada; $Y$
4. Aceptó asumir responsabilidad por todos los aspectos del estudio, asegurando que cada pregunta que surja en relación con la exactitud o integridad de cualquier parte del trabajo será investigada apropiadamente y resuelta por el grupo de autores.

Muchas revistas médicas exigen que cada autor explicite su participación en el estudio y en el manuscrito. Hace algunos años se preconizó en reuniones internacionales (en idioma inglés) sustituir el título de "author" por "contributor", describiendo el rol que había cumplido cada uno de los "contributors". Tuve la oportunidad en el ICMJE de votar en contra de este cambio, por dos razones. Primera: el concepto de "autor" tiene raigambre histórica y connotación sociológica respetables, que no estarían reflejadas en el término propuesto. Segunda: la traducción de "contributor" al idioma español ("contribuyente") tendría -al menos en Chile- una connotación peyorativa incómoda.

En las recomendaciones internacionales se explicitan formas para presentar la multiautoría, que en algunas áreas científicas implica acreditar a decenas o aun centenas de autores y unidades de trabajo (ejemplo: en física teórica). En medicina, la multiautoría es una situación creciente debida al aumento de las investigaciones colaborativas nacionales o internacionales. Al redactar su manuscrito, los autores principales deben seleccionar quienes figurarán como autores y quienes deben ser reconocidos como colaboradores, separando su ubicación en la página del título o en la sección "Agradecimientos". Tienen también la opción de identificar a dos personas que deben ir en primer lugar en la lista de autores, reconociendo que ambas lo merecen por haber contribuido con iguales méritos en la ejecución del trabajo y del manuscrito. Aquí aplica otro antiguo adagio: "Todos los que están, deben ser; todos los que son, deben estar."

Hay dos condiciones que suelen afectar la solvencia del listado de autores de un manuscrito: la inclusión de autores "honorarios" y la exclusión de autores "fantasmas". Autores "honorarios" suelen ser personas incluidas en la nómina porque son autoridades respetadas del sitio de trabajo y sus nombres podrían dar más peso o credibilidad a lo que se describe o afirma en el manuscrito -aunque no hayan participado en el estudio- o 
bien pertenecen a una unidad o departamento cuyos miembros pretenden -injustamente- figurar como autores en todas las publicaciones generadas allí. Autores "fantasmas" son personas que realmente participaron en el estudio, pero que se estimó prudente excluirlas de la nómina porque tienen conflictos de intereses tan obvios que podrían afectar la credibilidad del contenido y la intención del manuscrito (ejemplo: funcionarios de la organización que financió el estudio o que se beneficiará con la publicación de sus resultados).

En un artículo editorial en la Revista Chilena de Pediatría, J. O. Valderrama ilustró la magnitud de las faltas a los criterios de autoría, registrada en revistas con gran prestigio internacional ${ }^{25}$.

\section{Delitos o "felonías", enlistados en orden decreciente de gravedad}

- Invención, manipulación o falsificación de datos.

- Plagio de todo o partes del texto, o de datos pertenecientes a otro manuscrito.

- Publicación duplicada o redundante.

- No declarar oportunamente un conflicto de intereses.

- Autoría injustificada u oculta (autores "honorarios" y autores "fantasmas").

- Publicación fraccionada, "en rebanadas de salame" (salami slicing publication).

\section{Definición de plagio}

El Committee on Publication Ethics (COPE) define el plagio como "El uso de las ideas o las palabras de otros sin reconocerlo y sin su permiso, presentándolas como ideas originales y nuevas en vez de derivadas de una fuente ya existente. La intención y el efecto del plagio son engañar al lector en cuanto a las contribuciones del plagiador" 19 .

\section{Publicaciones duplicadas o redundantes}

Con la intención de aumentar el número de potenciales lectores de su manuscrito, hay autores que pretenden publicarlo simultánea o consecutivamente en distintas revistas, generalmente una general y otra especializada, una o ambas "de corriente principal". Esta intención se puede legitimar si se advierte a los editores de las revistas implicadas y se cumplen las recomendaciones del $\mathrm{ICMJE}^{18}$. Ellas empiezan por reconocer en la segunda revista la repetición de un mismo conjunto de datos, para evitar que sean sumados en caso de una revisión posterior del tema. Los manuscritos que informan sobre un problema emergente de salud pública o una nueva terapéutica con interés universal se someten a condiciones diferentes. La duplicidad autorizada debe declararse a las bases de datos, permitiendo su reconocimiento por quienes evalúen el número de publicaciones originales de los autores involucrados.

\section{Conflictos de intereses}

Existe conflicto de intereses cuando un participante en el proceso de publicación (autor, revisor o editor) tiene relaciones personales que podrían influir en este proceso o dar la apariencia de ello.

\section{Tipos de intereses competitivos:}

- Lazos financieros con una entidad que patrocinó el estudio.

- Competitividad académica/pasión intelectual.

- Relaciones interpersonales.

- Posiciones políticas o religiosas.

- Afiliación institucional.

Los conflictos de intereses más frecuentes tienen que ver con las relaciones de uno o más autores con sus entidades patrocinadoras: industria farmacéutica, fabricantes de equipos para diagnóstico o terapéutica (ejemplos: prótesis de caderas, marcapasos cardíacos, vacunas, implantes mamarios). Los autores pueden depender de la entidad patrocinadora en calidad de funcionarios, asesores, socios, accionistas, o recibir invitaciones para viajar, participar en congresos u otras reuniones, dar conferencias promocionales, etc.

Otra fuente importante de conflictos de intereses reside en la intervención de la entidad patrocinadora en la génesis del protocolo del estudio; en el análisis de los datos obtenidos (por ejemplo: cálculos estadísticos); en la redacción del manuscrito, enfocándolo en los resultados que estimen más convenientes y no en el propósito original del estudio ni en su resultado "outcome" primario; en la decisión de publicar o no el manuscrito y en la elección de la revista a la que será enviado.

Al enviar su manuscrito a una revista los autores deben adjuntar una declaración individual de potenciales conflictos de intereses. Las revistas adscritas a las recomendaciones del ICMJE pueden recurrir a un formato estándar, que se obtiene de su página $w_{e} b^{18}$.

Los revisores de manuscritos y los editores, a su 
vez, deben advertir cuando tienen una relación con los autores, académica, laboral o familiar; celos profesionales o académicos al competir en el tema del manuscrito; relaciones con una entidad en competencia con la que patrocina el manuscrito o cuyos intereses coinciden; o una posición religiosa, política o filosófica en pugna con la orientación del manuscrito. En tales circunstancias los revisores deben declinar la revisión y dar a conocer dichos conflictos de intereses a los editores. Los revisores externos y los editores adquieren el compromiso de no utilizar la información contenida en los manuscritos para fines personales y no deben divulgar sus aspectos originales hasta que se hayan publicado.

Si un editor tiene conflictos de intereses con un manuscrito (ejemplos: es coautor, o es autoridad académica en relación directa con los investigadores) deberá ser excluido de todo el proceso de revisión editorial del manuscrito, el que debe ser encargado a un coeditor que será responsable, con independencia total, desde la elección de revisores externos hasta decidir si se publica o no. En las revistas manejadas con programas digitales on line (en internet), es fácil excluir a un editor del acceso a la información correspondiente.

Declarar conflictos de intereses no implica reconocer una falta, porque no necesariamente han distorsionado el manuscrito o la opinión de los revisores. Los editores y los revisores externos pueden juzgar la posible influencia de los conflictos de intereses declarados por los autores, o descubiertos durante la revisión. Los editores deben facilitar a los revisores y los lectores el acceso a la declaración de conflictos de intereses de los autores. Los lectores pueden juzgar la idoneidad del manuscrito mediante esta información, tal como deben declararla quienes dictan una conferencia o participan en una mesa redonda. Esta medida es indispensable para la transparencia que deben tener las intervenciones de médicos y científicos, dado que pueden influir en la práctica profesional de los lectores y los asistentes a las reuniones científicas. Ello se resume en otro adagio: "Tener conflictos de intereses no es pecado: El pecado es no declararlos".

\section{Comentarios finales}

Desde 1997, en la Revista Médica de Chile se han detectado casos de publicaciones redundantes, de plagio, y de ocultamiento de conflictos de intereses. La mayoría de ellos fueron advertidos por acuciosos revisores externos. La falta más grave fue la falsificación de un manuscrito que había sido rechazado y que posteriormente tuvo una distribución restringida al grupo de autores, en un texto en formato "PDF" que remedaba el formato de la Revista. Ello despertó sospechas en uno de los coautores y lo advirtió a los editores, quienes verificaron el fraude y lo denunciaron a la institución de origen del manuscrito. Terminada la investigación institucional, la única persona identificada como responsable del delito renunció a su cargo.

Al descubrir una posible transgresión a la ética de las publicaciones científicas, los editores deben iniciar una investigación cuyo curso está protocolizado, para cada tipo de transgresión, en la base de datos $\mathrm{COPE}^{19}$. A los editores no les corresponde hacer una investigación exhaustiva ni proponer una sanción, porque "no son policías ni jueces". Ello es responsabilidad de las instituciones que patrocinaron el estudio y donde pertenecen los autores. Si el manuscrito aún no está publicado, el proceso editorial se debe detener hasta que haya un fallo por la institución involucrada. Cualquier intento de fraude crea un ambiente de desconfianza frente a futuros manuscritos de esos autores 9 .

"Lamentablemente, no se ha descubierto una fórmula que proporcione un escudo impenetrable para las faltas a la ética de las publicaciones científicas. La honestidad y buena fe de todos los actores del proceso (autores de manuscritos, sus revisores, los editores de las revistas) son indispensables para mantener el respeto que merecen quienes leen nuestras revistas, así como para el auto-respeto de cada uno de los participantes, porque las situaciones de mala conducta en las publicaciones científicas son faltas a la ética individual de los integrantes de los grupos involucrados y dañan el prestigio de las instituciones que sustentan su trabajo".

Aunque estas aseveraciones no hayan cambiado en los últimos años, el interés por comunicar el resultado de investigaciones científicas y observaciones clínicas originales sigue siendo un impulso legítimo y una actividad prioritariamente honesta y laudable. El progreso de las ciencias y la medicina dependen de ello. 


\section{Referencias}

1. López GS, Valdés GS, Roessler E, Valdivieso V. Declaración de la Academia Chilena de Medicina sobre el Título V de la Ley 20.850 (Ley Ricarte Soto) y su proyecto de reglamento "De los ensayos clínicos de productos farmacéuticos y elementos de uso médico". Rev Med Chile 2017; 145 (3): 380-5.

2. Reyes H. Ética de las publicaciones científicas. En: Reflexiones sobre bioética: Seminarios de la Academia Chilena de Medicina (2011-2013), págs. 120-8. Mireya Bravo (Compiladora). Academia Chilena de Medicina. CyC Impresores Ltda. Santiago, 2015.

3. Reyes H. Una publicación redundante en la Revista Médica de Chile. Rev Med Chile 1997; 125: 956-7.

4. Reyes H, Palma J, Andresen M. Actualización de los criterios de autoría en las publicaciones biomédicas (Editorial). Rev Med Chile 2002; 130: 1083-6.

5. Reyes H, Palma J, Andresen M. La importancia de declarar un conflicto de intereses en las revistas médicas (Editorial). Rev Med Chile 2003; 131: 7-9.

6. Editores. El conflicto de intereses en las revistas biomédicas (Artículo Especial). Rev Med Chile 2003; 131: 93-4.

7. Declaración conjunta de la Federación Europea de Medicina Interna, el American College of Physicians-American Society of Internal Medicine y el American Board of Internal Medicine (Ética Médica). Profesionalismo médico en el nuevo milenio: Un estatuto para el ejercicio de la medicina. Rev Med Chile 2003; 131: 457-60.

8. Reyes H, Palma J, Andresen M. Ética de las publicaciones en revistas médicas (Artículo Especial). Rev Med Chile 2007; 135: 529-33.

9. Reyes H. Honestidad y buena fe: dos pilares en la ética de las publicaciones biomédicas (Editorial). Rev Med Chile 2007; 135: 415-8.

10. Comisión de Ética de la Facultad de Medicina de la Universidad de Chile. En referencia al plagio intelectual (Documento). Rev Med Chile 2008; 136: 653-8.

11. Reyes H. El plagio en publicaciones científicas (Editorial). Rev Med Chile 2009; 137: 7-9.

12. López R. Crear o copiar... ¿Cuál es la diferencia? (Artículo Especial). Rev Med Chile 2009; 137: 121-6.

13. Drazen JM, De Leeuw PW, Laine C, Mulrow C, DeAngelis CD, Frizelle FA, et al. Toward More Uniform
Conflict Disclosures - The Updated Reporting Form for Disclosure of Potential Conflicts of Interest (Editorial). Rev Med Chile 2010; 138: 801-3.

14. Valenzuela CY. Ética científica y uso de material o datos recolectados de seres humanos (Ética Médica). Rev Med Chile 2012; 140: 390-5.

15. Soto Subiabre M. Declaración de conflictos de intereses: contribución a la mejoría en la calidad ética de las publicaciones. Rev Med Chile 2016; 144 (11): 1067-72.

16. Von Oetinger A, Sadarangani KP, Salas SP. Conflictos éticos en las autorías de trabajos científicos. Rev Med Chile 2016; 144 (8): 1473-8.

17. Portales MB, Michaud P, Salas SP, Beca JP. Formas de revisión ética de proyectos de investigación médica. Rev Med Chile 2017; 145 (3): 386-9.

18. International Committee of Medical Journal Editors. Recommendations for the Conduct, Reporting, Editing, and Publication of Scholarly work in Medical Journals. Updated December 2017. Accesible en www.icmje.org/ recommendations/

19. Committee on Publication Ethics. Principles of Transparency and Best Practice in Scholarly Publishing Version 3, January 2018. Accesible en https://publicationethics. org/resources/guidelines.

20. World Association of Medical Editors (WAME). Accesible en www.wame.org

21. Day RA, Gastel B. Cómo escribir y publicar trabajos científicos. Organización Panamericana de la Salud, Publicación Científica y Técnica No 621, 6a Edición, 2008.

22. The Office of Research Integrity. US Department of Health \& Human Services. Accesible en: https://ori.hhs. gov

23. Valenzuela MT, Urquidi C, Martínez C. Manual para la estandarización de estudios clínicos en Chile: desde la concepción hasta la publicación. Universidad de los Andes, Santiago, 2017. Accesible en: www.ispch.cl/anamed

24. Valdés GS. Requisitos actuales de la investigación experimental en animales: realidades y reflexiones. Boletín de la Academia Chilena de Medicina, No LIII, 2016, págs. 99-109, Manuel Oyarzún (Editor). Gráfica LOM, Santiago, 2017.

25. Valderrama JO. Aspectos éticos en las publicaciones de revistas científicas de corriente principal (Editorial). Rev Chil Pediatr 2012; 83 (5): 417-9. 